\title{
Just in the nick of time
}

\section{Targeting acute versus chronic disease}

\section{Kenneth Maiese}

Editor-in-Chief; Professor and Chair; Department of Neurology and Neurosciences; University of Medicine \& Dentistry of New Jersey; New Jersey Medical School; Newark, NJ USA

The statement "timing is everything" in many instances can mean the difference between successful outcomes and tragic results in the business, sports and entertainment industries. Multiple variables and environmental factors can significantly impact outcomes in relation to the time that they occur. Yet, "timing" or the sequence of events are probably just as critical for cellular biological processes that ultimately lead to many diseases whether they are considered acute or chronic in nature. If one considers the nervous system, the events that lead to the immediate onset of a stroke in an individual also may be the same processes that result in more chronic disability, such as occurs during Alzheimer's disease. For example, recent work suggests that proteins derived from the Drosophila Wingless $(\mathrm{Wg})$ and the mouse Int-1 genes may be associated with several disease entities. Wnt proteins are secreted cysteine-rich glycosylated proteins that can control cell proliferation, polarity, longevity and tumorigenesis. These genes are present in several cellular populations including neurons, cardiomyocytes, endothelial cells and cancer cells. Interestingly, Wnt proteins may control the acute stages of metabolic disorders such as glucose tolerance and insulin sensitivity. However, abnormalities in the Wnt pathway also lead to increased risk and progression to type 2 diabetes mellitus, suggesting that the early onset of impaired Wnt signaling may initiate and progress to more chronic disability. Evidence for such a scenario with Wnt is seen with altered Wnt signaling tied to acute stroke injury, the rapid development of malignant neoplastic disease and the potential onset of other long-term neurodegenerative disorders that include Alzheimer's disease. As a result, targeting Wnt and novel pathways controlled by the Wnt family may offer novel therapeutic means that address in a timely manner the onset and progression of acute as well as chronic disorders.

In this issue of Oxidative Medicine and Cellular Longevity, our papers shed further light on a number of cellular targets that control both acute and long-term disorders, suggesting that one significant variable to address is the onset and timing of these processes. Igor Afanas'ev in an opinion paper provides new insights for the potential role of reactive oxygen and nitrogen species in diabetes mellitus. He focuses upon several transcriptional pathways as well as advanced glycation end-products in the ability of these agents to accelerate cell injury and complications of diabetes through enhanced oxidative stress, suggesting that early therapeutic intervention of these pathways may prevent both acute neuronal and vascular injury during diabetes but also block the progression of debilitating chronic injury. In their review paper, Chong et al. demonstrate for us how a single but central pathway can determine the onset and progression of not only acute injury, but also chronic disease processes. These authors offer a critical analysis of new work on the mammalian target of rapamycin (mTOR) and provide justification for the early manipulation of this pathway in multiple disorders since mTOR impacts a spectrum of acute and chronic neurodegenerative disease processes such as stroke, trauma, Huntington's disease, Parkinson's disease, epilepsy, Alzheimer's disease and tuberous sclerosis. In our second review paper for this issue, Yang et al. analyze forces that play upon the blood retinal barrier during metabolic disorders and illustrate the role of cytokines, growth factors and cellular pathways of inflammation that can have a concerted effect on acute and long-lasting injury pathways. Attia et al. in their original work target the novel use of proanthocyanidins to elucidate for us the potential efficacy of such therapy to prevent the early and late effects of doxorubicin toxicity on germinal cell lines. Das and Mukherjee take this concept of elucidating early cellular changes in disease models one step further to identify potential strategies against chronic ethanol exposure by illustrating the role and timing of elevated oxidant stress pathways that can precipitate lung tissue injury. Next, Canever et al. provide an extremely intriguing study that describes in an experimental model for schizophrenia the link of creatine kinase to behavior changes, suggesting that the early onset of alterations in cerebral metabolism may result, under some scenarios, in the onset of a complex chronic disorder such as schizophrenia. Alshabanah et al. delve further into toxic cellular pathways and chemotherapeutic agents to not only examine the contribution of oxidative stress to cell injury, but also to identify unique treatment strategies with L-carnitine, an endogenous mitochondrial membrane component, that can prevent hepatic injury during doxorubicin administration. Our final article for this issue by Venturini et al. is no less fascinating than our other studies. The work of these authors identifies both red wine and resveratrol as acute acting antioxidants in the brain during experimental diabetes but that the principal effects of red wine appear limited to the hippocampus while the protective effects of resveratrol are more widespread and affect both hippocampal and cortical regions. Thus, the studies in this issue of Oxidative Medicine and Cellular Longevity serve to 
provide us with greater understanding of the cellular pathways and treatment targets that can be considered for acute and chronic disorders. However, possibly more critical is the knowledge gained from the work in this issue that potentially the only critical difference between acute and chronic disease processes is not their onset or progression, but rather the necessary therapeutic intervention that is required "just in the nick of time" to avert disease onset and disability. 


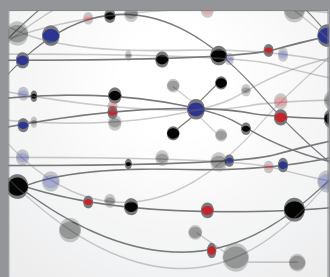

The Scientific World Journal
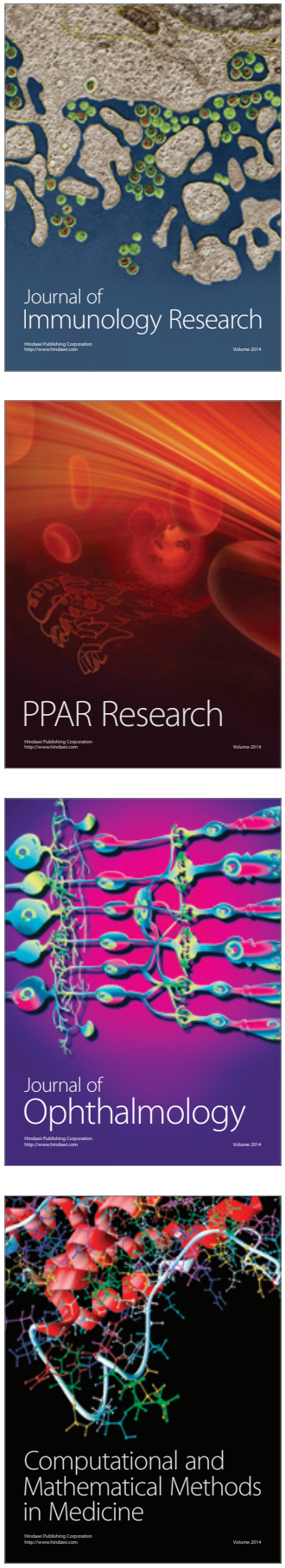

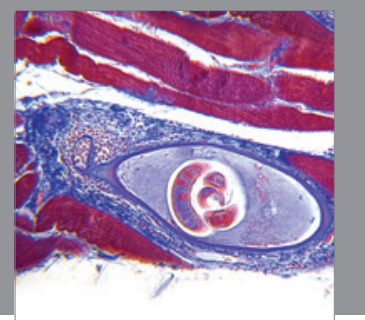

Gastroenterology

Research and Practice
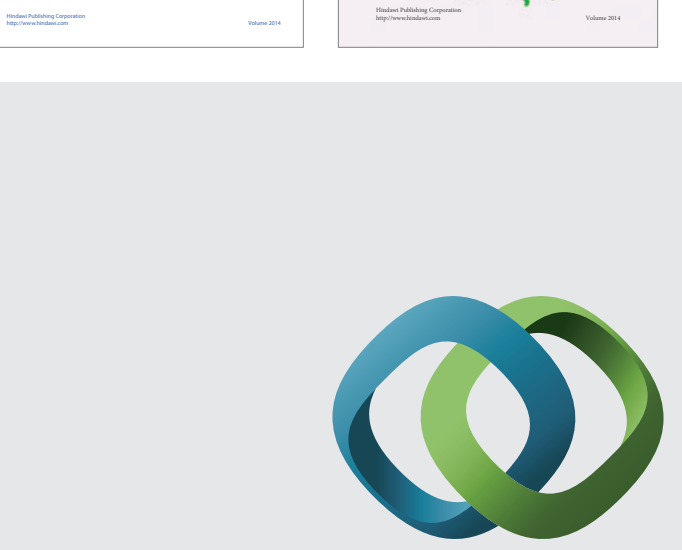

\section{Hindawi}

Submit your manuscripts at

http://www.hindawi.com
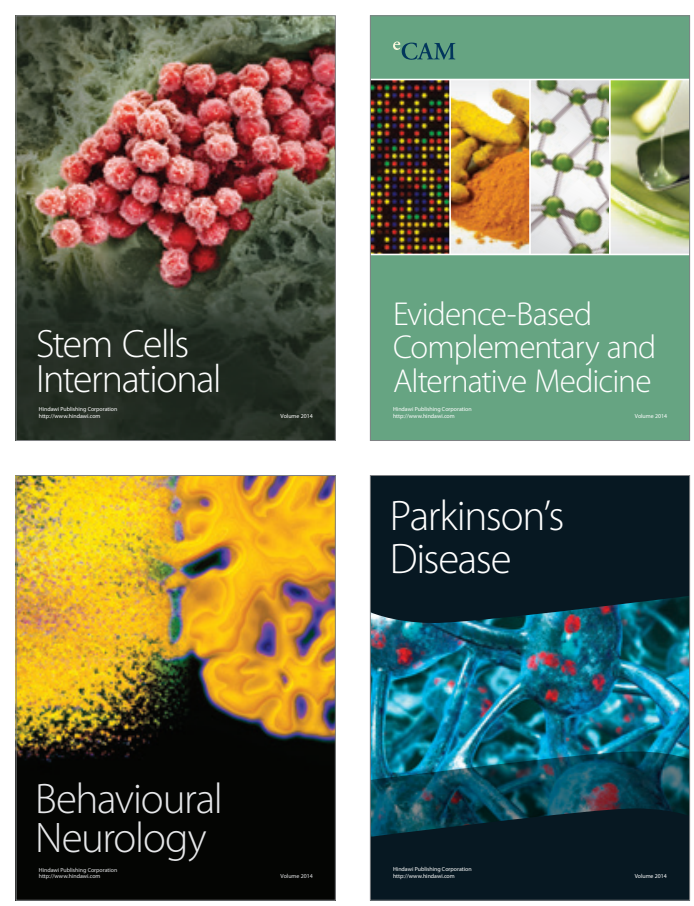

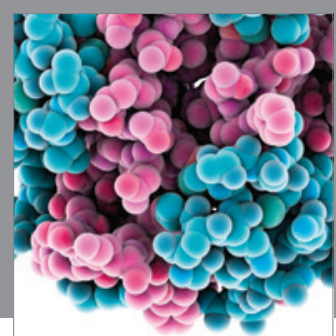

Journal of
Diabetes Research

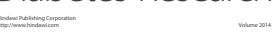

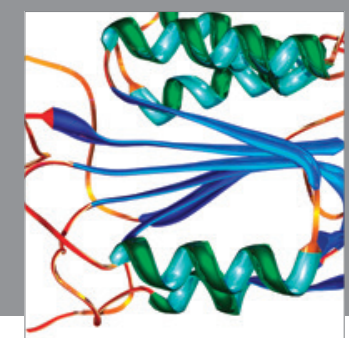

Disease Markers
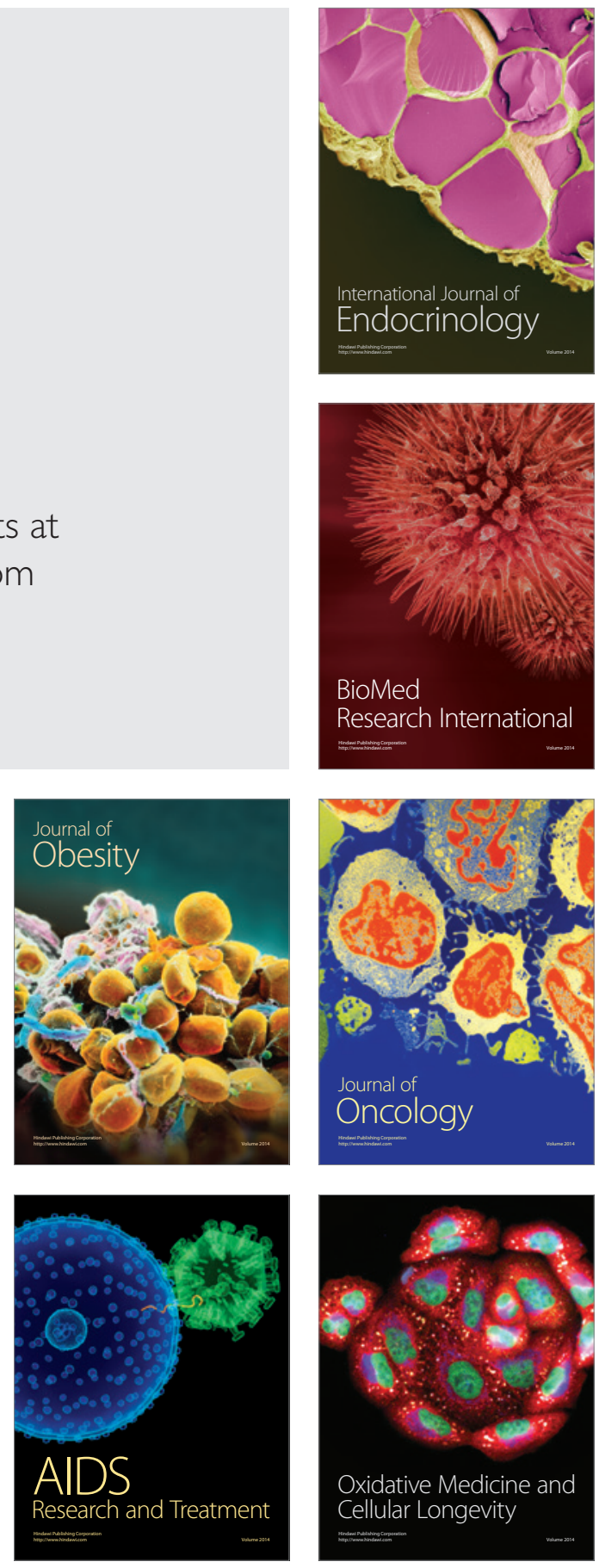